\title{
Dimensional Analyses of Geometric Products and the Boundary Condi- tions of the Universe: Implications for a Quantitative Value for the La- tency to Display Entanglement
}

\author{
Michael A. Persinger ${ }^{*}$ and Stanley A. Koren
}

Laurentian University, Sudbury, Ontario, P3E 2C6, Canada

\begin{abstract}
We explored the dimensional equivalence of the product of the plane, area, and volume of a circle as well as frequency $(2 \pi \mathrm{rf})$, or $21.3 \pi^{4} \mathrm{r}^{7} \mathrm{f}$, when applied to universal boundaries. When this product was set equal to the equivalent powers of the gravitational constant and the mass, age, and width of the universe a "diffusion" velocity of $\sim 10^{23} \mathrm{~m} \cdot \mathrm{s}^{-1} \mathrm{was}$ derived which matched the $\sim 7$ to 8 min associated with non-locality and "entanglement" as indicated by both theoretical estimates for gravitational solitons and empirical inferences for photon coupling. These results suggest that a recondite factor employed to describe a closed geometry (the circle) in three dimensions and time (as frequency) may offer alternative perspectives to quantitative solutions for penetrance of multidimensional space within submass levels. Latency to display universal entanglement may not be instantaneous or continuous but would involve a discrete quantitative value that could have significant implications for physical cosmology.
\end{abstract}

Keywords: Nonlocality, entanglement, complex geometry, solitons, fractals, quantitative solutions, universal "diffusivity".

\section{INTRODUCTION}

Mathematical descriptions of an infinite but closed geometry, the circle, is the basis of our assumptions and perceptions concerning the physical world. The quintessential single dimensional circle describes the orbit of electrons, horizons of universal boundaries and the singularity. A soliton can be viewed as a propagating wave with selfreinforcing components (a pulse or wave packet) because of a changing angular velocity where the group and phase velocity are dissociated. The closed loop is also the simplest representation of self-similar patterns or fractals that emerge as orbital patterns for electrons, planets and even stars around galactic centers. When the derivates are included from circles expressed in space and time, the fractal dimensions can be considered greater than the topological dimensions. Such complexity has the potential to be revealing with respect to relating the challenges of relating the properties of space with the physical parameters that define universal boundaries.

In the classical four dimensional (Kantian) world composed of three spatial axis and one temporal plane, the representations of a closed but infinite geometry would be $2 \pi \mathrm{r}$ (for the perimeter of a circle), $4 \pi r^{2}$ (for the surface of a sphere), $4 / 3 \pi r^{3}$ (for the volume of a sphere), and $2 \pi r f$ for the time component, where $\mathrm{f}=$ frequency. We assumed, for the purpose of quantitative exploration, that these universal descriptions are components of a larger aggregate derived from multiplicative combinations. The product of these relationships

*Address correspondence to this author at the Laurentian University, Sudbury, Ontario, P3E 2C6, Canada; Tel: 01-705-675-4824;

Fax: 01-705-671-3844; E-mail: mpersinger@laurentian.ca is $21.3 \pi^{4} \mathrm{r}^{7} \mathrm{f}$ which results in the higher dimensional aggregate of $\mathrm{m}^{7} \mathrm{~s}^{-1}$. As suggested by Konstantinov [1] consideration of four dimensional space-time as a submanifold embedded into a Euclidean space of sufficiently high dimensions may be a valid method to solving the topology of the spatial sections and their transitions within the global structure [1].

We then explored the implications of this metric when applied to the essential parameters employed to define the known universe. The resulting solutions may be relevant to entanglement and fractal-like quantitative relationships between levels of spatial organization. Although in our analyses we employed coefficients to two decimal points for convention, the current errors of estimation and measurement suggest that the integer and power of 10 for values that define the entire set (the boundary) are likely to be most representative.

\section{THE MODEL}

The assumption that the ultimate geometry of the universe should be consistent with its temporal, spatial and energetic-mass relations is an implicit component to many approaches to physical cosmology [2-4]. If the units of geometric descriptions are set equal to physical "constants" that define the set, in this case the universe, then solutions should emerge that are internally consistent with other measurements. These new solutions may also reveal occluded values that could reflect parameters by which the entire set is intrinsically organized. A solution from dimensional analysis that results in $\mathrm{m}^{7} \mathrm{~s}^{-1}$ would be $\left[\mathrm{m}^{6} \mathrm{~kg}^{-2} \cdot \mathrm{s}^{-4}\right] \cdot \mathrm{kg}^{2} \cdot \mathrm{m}^{3} \mathrm{~s}^{3}$.

One combination of values that would also reflect the essential current properties of the universe would be $\mathrm{G}^{2} \cdot \mathrm{m}^{2} \cdot \mathrm{d} \cdot \mathrm{t}^{3}$, such that: 
$21.3 \pi^{4} \mathrm{r}^{7} \mathrm{f}=\mathrm{G}^{2} \cdot \mathrm{m}^{2} \cdot \mathrm{d} \cdot \mathrm{t}^{3}$

where $G$ is the Newtonian gravitational constant, $m=$ the mass of the universe, $\mathrm{d}=$ the diameter of the universe, and $\mathrm{t}=$ the age of the universe. The mass of the universe was inferred by obtaining the equivalent metric between $G$ and universal pressure $\left(\partial \mathrm{c}^{2}\right)$, where $\partial=$ average density and $\mathrm{c}$ is the velocity of light in a vacuum [5]. The estimated value is $2.38 \cdot 10^{52} \mathrm{~kg}$. This mass is within the range obtained empirically assuming the average star displays a solar mass of $\sim 10^{30} \mathrm{~kg}$, there are $10^{11}$ stars per galaxies and there are a total of $10^{11}$ galaxies. The radius to the horizon (boundary) can be estimated by:

$f=8 \pi \mathrm{G} / \mathrm{c}^{2}$

which results in the constant of $1.86 \cdot 10^{-26} \mathrm{~m} \cdot \mathrm{kg}^{-1}$. When this value is multiplied by $2.38 \cdot 10^{52} \mathrm{~kg}, \mathrm{r}=4.43 \cdot 10^{26} \mathrm{~m}$ and the width is $8.86 \cdot 10^{26} \mathrm{~m}$. To our knowledge a solution where this particular geometric assumption $\left(21.3 \pi^{4} \mathrm{r}^{7} \mathrm{f}\right)$ has been balanced with measurements of the properties of the entire set, the universe, has not been pursued for novel solutions.

\section{EMERGENT SOLUTION}

The quantitative solution for $\mathrm{m}^{7} \mathrm{~s}^{-1}$ yields a "hyperdimensional" value for the entire universe's boundary for intrinsic rates of dispersion. When the actual values: $6.67 \cdot 10^{-11} \mathrm{~m}^{3}$ $\mathrm{kg}^{-1} \mathrm{~s}^{-2}$ for $\mathrm{G}, 2.38 \cdot 10^{52} \mathrm{~kg}$ for the universal mass, 8.86 . $10^{26} \mathrm{~m}$ for the universe's width, and $4.06 \cdot 10^{17} \mathrm{~s}(13$ billion years) for its duration are inserted into (1), the value is 1.49 . $10^{164} \mathrm{~m}^{7} \mathrm{~s}^{-1}$. The $7^{\text {th }}$ root, which would reflect the "diffusion" of this hypothetical "quality", would be $2.84 \cdot 10^{23} \mathrm{~m} \mathrm{~s}^{-1}$. Such quality "diffusing" at this velocity in today's universe of $1.22 .10^{26} \mathrm{~m}$ (the product of its age, c, and $\mathrm{s} \cdot \mathrm{yr}^{-1}$ ) would require $4.30 \cdot 10^{2} \mathrm{~s}$ or between 7 and 8 minutes to pervade its extent. It is relevant that other assumptions of the mass of the universe $\left(1 \cdot 10^{52} \mathrm{~kg}\right)$ that accommodate dimensionless parameter $A$ and other shape-relating values $[2,5,6]$ that assume the circumference of the horizon rather than its diameter $\left(11.68 \cdot 10^{26} \mathrm{~m}\right)$ do not appreciably change the "diffusion" value between 7 and $8 \mathrm{~min}$.

\section{IMPLICATIONS AND APPLICATIONS}

Reciprocal changes in the properties of paired, entangled photons have been assumed to be "instantaneous" even at opposite ends of the universe [7]. This assumption may reflect the limited scope of contemporary measurement. For example the dissociative reaction associated with chemical bonds after absorption of a photon was also considered "instantaneous" and thus immeasurable until Rosker et al. [8] demonstrated the discrete interval of bond persistence in the order of $10^{-13} \mathrm{~s}$ by femtosecond laser techniques. Pursuit of the mechanisms and processes responsible for this discrete interval have been revealing with regards to the concepts of microentanglement. A discrete value that represents the "diffusion" of processes over the spatial extent of the universe within 7 to 8 min may also have application to entanglement.

One of the counterintuitive consequences of a holographic universe is that each of the smallest points must be mapped onto the whole and it must be mapped to the each of the smallest points [9]. Persinger and Koren [10] showed the cosmological implication of this problem. First, Hubble's constant $\sim 10^{-18} \mathrm{~s}^{-1}$ was multiplied by the largest distance (width of the universe) or the smallest distance (Planck's length). These values were then divided into Planck's constant to obtain duration (time). They found that the time required for Planck's length to expand or "jump" one Planck's length would require $10^{17} \mathrm{~s}$, or the age of the universe. The time for the universal width to expand one Planck's length would be $10^{-44} \mathrm{~s}$ or Planck's time. What is clear is that the largest space (the universe) and the smallest space (Planck's length) converge to some limit where the characteristics of one approach those of the other. From the perspective of classical concepts of non-locality, "instantaneousness" for these "spooky actions at a distance" has been a presumption without a clear metric.

However the duration of 7 to $8 \mathrm{~min}$ for a process that diffuses through the multidimensional levels of space within the universe may not be spurious in light of both theoretical and empirical solutions concerning possible macroscopic manifestations of non-locality and entanglement. According to $\mathrm{Tu}$ et al. [11] one of the consequences of a photon displaying a non-zero mass is that the phase and group velocities would be dissociated. The dispersion of photons within the $10^{8}$-to $10^{15} \mathrm{~Hz}$ range (that includes visible light) would display a difference in velocity $(\Delta \mathrm{c} / \mathrm{c})<10^{-7}$. When inserted into the classical Lorentz contraction of:

$t_{\Delta}=1 / \sqrt{ } 1-\left(\mathrm{v}^{2} / \mathrm{c}^{2}\right)$

the difference would be $\sim 8 \mathrm{~min}$.

The quantum efficiency for two volumes of caesium gas described by Julsgaard et al [12] involved temporal parameters between 7 to $8 \mathrm{~min}$. Recently Dotta et al. [13] systematically replicated results that strongly suggested macroentanglement by experimentally dissociating the group and phase velocities of weak rotating magnetic fields, in accordance with $\mathrm{Tu}$ et al's. [11] theoretical solutions. Dotta et al. [13] generated computer-produced patterns of magnetic fields with asymmetric angular accelerations and decelerations within two separate circular arrays of solenoids. Chemical reactants were placed in the center of each array. Simultaneous initiation of photochemical reactions (hydrogen peroxide injected into a solution of sodium hypochlorite) in these two loci separated by $10 \mathrm{~m}$ produced a conspicuous doubling of photon emissions as if the two separate loci were superimposed into one space. However the duration of this visually obvious "doubling" effect in photon yield, which we recently verified over $3 \mathrm{~km}$, diminished after 7 to 8 minutes even though the same reactions were continued. The effect did not occur if, as predicted by Tu et al. [11] the phase and group velocities of the applied fields were not dissociated or if the optimal sequence of the patterns were reversed.

We had considered that the "doubling photon" effect with its transient existence of $\sim 8$ min could be explored as events within quantum gravity Hilbert space and as a possible extended particle analogous to a soliton [14]. El Naschie [15] deduced that the energy equivalent of the mass of the fundamental exotic transfinite particle would be $\sim 3 \cdot 10^{-13} \mathrm{~J}$ in standard settings. This value is within less than an order of magnitude of the values we observed as incremental increases in photon emissions during our non-locality paradigm [13]. Gravitational instantons are conceived as tunneling events where there is a sudden appearance of a micro- 
scopic window in space-time. The $7^{\text {th }}$ root of spatial dimensions could satisfy the highly local nonlinearity required for simple models of solitons [16]. Although perhaps spurious the mass for El Naschie's exotic transfinite particle when the derived energy is divided by the cross-sectional area of the universe and multiplied by the square of its age is the same order of magnitude as the mass of an electron. In addition, if the volume of the electron from the classical radius (assuming a sphere, $\sim 94 \cdot 10^{-45} \mathrm{~m}^{3}$ ) was composed fundamentally of a cross-sectional area derived from Planck's length $\left(8.24 \cdot 10^{-}\right.$ ${ }^{70} \mathrm{~m}^{2}$ ) folded within hyperdimensional geometry, the length must be $\sim 1.14 \cdot 10^{26} \mathrm{~m}$, that is, approaching the estimated radius of the universe, to complete this volume. Such properties would be expected from a condition connecting the smallest to the largest space.

If the discrete value for "diffusion" is valid, then application to specific spaces should be consistent with what has been measured at these levels of discourse. This assumption embodies the concept of macrocosm-microcosm, the fractal and the gnomon [17]. The latter is defined by its intrinsic replicative reiteration at larger or smaller levels of spatial organization relative to the reference of observation. As tests of application, we selected two important spaces for astronomy: the circumference of the earth and the approximate radius of the solar system.

The time required for the $7^{\text {th }}$ dimensional diffusion of the proposed entanglement or non-locality quality to traverse the circumference of the earth $\left(4 \cdot 10^{7} \mathrm{~m}\right)$ is about $1.4 \cdot 10^{-16} \mathrm{~s}$ which is approaching the single orbital time for the Bohr magneton $\left(1.5 \cdot 10^{-16} \mathrm{~s}\right)$. This discrete duration serves as a component of the bases for the value of Planck's constant (the product of the mass of an electron, the square of the fine-structure velocity and $1.5 \cdot 10^{-16} \mathrm{~s}$, for $\mathrm{J} \cdot \mathrm{s}$ ) upon which quantum solutions for energy depend. If the "diffusion" value derived from (2) reflects an intrinsic entanglement, then the time required for one orbit of the (Bohr) electron would be the discrete increment required for this phenomenon to occur.

For larger perimeters, such as the solar system, the diffusion value predicts the significance of $10^{-12} \mathrm{~s}$ for intrinsic entanglement. This is exactly the range of duration of the hydronium $\left(\mathrm{H}_{3} \mathrm{O}^{+}\right)$ion whose empirical values range from 0.65 to $3 \mathrm{ps}$ [18]. Although only one proton in a billion is a component of a hydronium ion at any $\Delta t$, the continuous change indicates that all water molecules within the shared volume will be exposed to different protons that composed the "same" structure. The relatively small annual changes in linear distance between the sun and the earth as a function of orbital position should be reflected in small but discernable seasonal periodicities of aqueous chemical reactions within fixed volumes. There is evidence for this possibility [18-20].

\section{CONCLUSIONS}

The solutions presented here, derived from equivalence between the multiplicative combination of the fundamental descriptions of a singularity (a circle) in four-dimensional space-time, predict that there may be an intrinsic "diffusion" time or latency within the volume of space defining the present universe. Although this "diffusivity" value exceeds c, the quantity might be applied to the concept of "entanglement". It might not be instantaneous but within universal boundaries would require durations in the order of 7-8 $\mathrm{min}$. The realization that the velocity of light was not instantaneous altered astronomical perspectives. A discrete value for entanglement derived from quantitative values that reflect the entire universe could offer an alternative explanation to "instantaneous non-locality" or "spooky actions at distance". At smaller spaces the solution overlaps with fundamental discrete durations that could couple essential physical chemical reactions with this universal feature of "diffusivity". This connection could encourage a reconsideration of Ernst Mach's late $19^{\text {th }}$ century principle of the imminence of the universe which indicates that properties of local matter depend upon the presence of the remainder of the universe. If this conclusion is valid and entanglement time does have a discrete and finite value, then an experiment could be potentially completed that could test this supposition.

\section{CONFLICT OF INTEREST}

The authors confirm that this article content has no conflicts of interest.

\section{ACKNOWLEDGEMENTS}

Declared none.

\section{REFERENCES}

[1] Konstantinov MY. Topological transitions and large-scale structure of space-time in multidimensional theory of gravity. Russ Phys J 1997; 40: 124-7.

[2] Persinger MA. Convergent calculations that dark solutions are reflective of mass-energy yet to occur. Int $\mathrm{J}$ Astron Astrophys 2012; 2: 125-8.

[3] Szydlowski M, Godlowski W. Acceleration of the universe driven by Casimir forces. Int J Mod Phys A 2008; 17: 343-66.

[4] Audretsch J. Reimannian structure of space-time as a consequence of quantum mechanics. Phys Rev D 1983; 27: 2872-83.

[5] Persinger MA. A simple estimate for the sass of the universe: dimensionless parameter A and the concept of 'pressure'. J Phys Astrophys Phys Cosmol 2009; 3: 1-3.

[6] Koren SA, Persinger MA. The Casimir force along the universal boundary: quantitative solutions and implications. J Phys Astrophys Phys Cosmol 2010; 4: 1-4.

[7] Aczel AD. Entanglement: the greatest mystery in physics. Vancouver: Raincoast Books 2001.

[8] Rosker MJ, Dantus M, Zewail AH. Femtosecond clocking of the chemical bond. Science 1988; 241: 1200-2.

[9] Di Base F. Quantum-holographic informational consciousness. Neuroquantology 2009; 7: 657-64.

[10] Persinger MA, Koren SA. A theory of neurophysics and quantum neuroscience: implications for brain function and the limits of consciousness. Int J Neurosci 2007; 117: 157-75.

[11] Tu LC, Lou J, Gilles GT. The mass of the photon. Rep Prog Phys 2005; 68: 77-130.

[12] Julsgaard B, Kozhekin A, Polszik ES. Experimental long-lived entanglement of two macroscopic objects. Nature 2011; 412: 4003.

[13] Dotta BT, Persinger MA. "Doubling" of local photon emissions when two simultaneous, spatially separated, chemiluminescent reactions share the same magnetic field configurations. J Biophys Chem 2012; 3: 72-80.

[14] Ambjorn J, Jurkiewicz J, Loll R. Emergence of a 4D world from causal quantum gravity. Phys Rev Lett 2004; 93: 13101-4.

[15] El Naschie MS. Gravitational instanton in Hilbert Space and the mass of high energy elementary particles. Chaos Solitons Fractals 2004; 20: 917-23.

[16] Synder AW, Mitchell DJ. Accessible solitons. Science 1997; 276: 1538-40.

[17] Gazale MJ. Gnomon. Princeton: Princeton University Press 1999.

[18] Decoursey TE. Voltage-gated proton channels and other proton transfer pathways. Physiol Rev 2003; 83: 475-579.

[19] Schnoll SE, Kolombet VA, Pozharskii, EV, Zenchenko TA, Zvereva IM, Kondradov AA. Realization of discrete states during fluc- 
tuations in macroscopic processes. Phys Uspekhi 1998; 41: 102535.

[20] Klocheck NV, Palamarchuk LE, Nikonova MV. Preliminary results of investigations into the effect of cosmophysical radiation of a non-electromagnetic nature of physical and biological systems. Biophysics 1995; 40: 883-91.

Received: December 03,2012

(C) Persinger and Koren; Licensee Bentham Open.

This is an open access article licensed under the terms of the Creative Commons Attribution Non-Commercial License (http://creativecommons.org/licenses/by-nc/3.0/) which permits unrestricted, non-commercial use, distribution and reproduction in any medium, provided the work is properly cited. 